\title{
What can neutron stars reveal about the equation of state of dense matter?
}

\author{
Ingo Tews ${ }^{1, \star}$ \\ ${ }^{1}$ Theoretical Division, Los Alamos National Laboratory, Los Alamos, New Mexico 87545, USA
}

\begin{abstract}
Neutron stars are astrophysical objects of extremes, reaching the highest densities we can observe in the cosmos, and probing matter under conditions that cannot be recreated in terrestrial experiments. In August 2017, the first neutron-star merger has been observed, which provided compelling evidence that these events are an important site for r-process nucleosynthesis. Furthermore, the gravitational-wave signal of such events might shed light upon the nature of strongly interacting matter in the neutron-star core. To understand these remarkable events, reliable nuclear physics input is essential. In this contribution, I explain how to use chiral effective field theory and advanced many-body methods to provide a consistent and systematic approach to strongly interacting systems from nuclei to neutron stars with controlled theoretical uncertainties. I will discuss recent results for the equation of state relevant for the nuclear astrophysics of neutron stars and neutron-star mergers.
\end{abstract}

\section{Introduction}

Neutron stars (NSs) are extreme stellar objects. They are born in core collapse supernovae of stars with about 8-20 solar masses [1] and, hence, are one of the final stages of stellar evolution. With typical masses of $1.4 M_{\odot}$ and radii of $10-14 \mathrm{~km}$, NSs reach densities comparable to nuclear saturation density, $n_{\text {sat }} \approx 2.7 \cdot 10^{14} \mathrm{~g} / \mathrm{cm}^{3}$, and maybe up to $\sim 10 n_{\text {sat }}$ in their core. Due to these high densities, typical temperatures are small compared to the Fermi energy, and NSs can be considered as cold, $T=0$. In addition to very high densities, NSs also explore large magnetic fields, high rotational frequencies, and extreme regimes of gravity.

Neutron stars consist of strongly interacting matter. Their outer crust is made up of a lattice of nuclei that become increasingly neutron-rich with growing density. At the so-called neutron-drip density, approximately $4 \cdot 10^{11} \mathrm{~g} / \mathrm{cm}^{3}$, the neutron chemical potential is so high that neutrons can exist outside of the nuclei. In this inner crust, the lattice of nuclei is surrounded by a neutron fluid. At the bottom of the inner crust, exotic pasta phases can appear, and nuclei form structures lie rods and slabs. At densities of roughly $0.5 n_{\text {sat }}$, the crust dissolves and NSs consist of a nuclear fluid in $\beta$ equilibrium, with roughly $95 \%$ neutrons and $5 \%$ protons. At even larger densities, above $2 n_{\text {sat }}$, NSs explore strongly-interacting matter that cannot be realized in terrestrial experiments. At these densities, exotic phases of matter, e.g., deconfined quark matter [2], might appear. Hence, NSs are ideal systems to study the dense-matter equation of state (EOS) and the QCD phase diagram at $T=0$.

^e-mail: itews@lanl.gov 


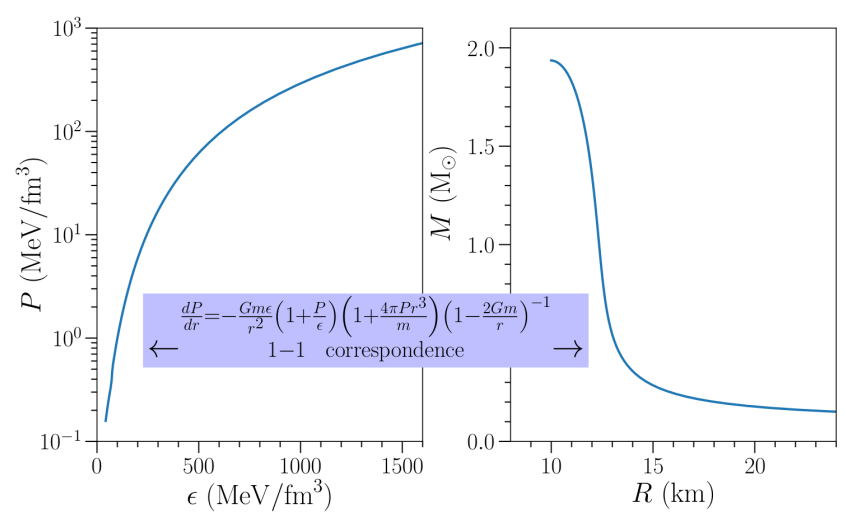

Figure 1. The EOS for NSs (left) and the resulting MR curve (right), for the Skyrme model NRAPR [3]. Figure taken from Ref. [7].

In this contribution, I will explain how we can use state-of-the-art nuclear theory and NS observations to unravel the EOS of dense matter.

\section{The equation of state}

\subsection{General considerations}

Neutron stars are described by the EOS, which is a relation among the energy density $\epsilon$, the pressure $p$, the temperature $T$ and the composition. Because individual NSs can be considered at $T=0$, the EOS is simplified ${ }^{1}$. With a given composition of NS matter, the EOS reduces to a simple relation $p(\epsilon)$. Given this relation, one can solve the Tolman-Oppenheimer-Volkoff equations and obtain the massradius (MR) relation of NSs. The MR relation and the EOS are in 1-to-1 correspondence, see Fig. 1 for an example EOS. As a consequence, NS observations of the MR relation, or even a single point of this curve, allow constraints on the dense-matter EOS itself. While NS masses can be determined quite precisely, radii are very difficult to determine [4]. Currently, the strongest observational constraints come from the existence of two-solar-mass NSs [5, 6], which allows to rule out too soft EOS (with lower pressure) that cannot sustain NSs of this mass against gravitational collapse. Radii, on the other hand, have large uncertainties and range from 10-14 km. This uncertainty range reflects the fact that the EOS at large densities (and even its composition) is unknown.

Theoretical calculations allow us to constrain the EOS and, hence, to improve our understanding of NSs and their properties. At low densities, nuclear theory provides constraints on the EOS and I discuss them in the following section. At very high densities about $50 n_{\text {sat }}$, well above neutronstar densities, EOS constraints can be obtained by perturbative QCD calculations [8,9], which are not discussed here. At intermediate densities, there are no robust theoretical EOS constraints, but NS observations of orbital characteristics in binaries, gravitational waves, or in the electromagnetic spectrum might fill this gap.

\footnotetext{
${ }^{1}$ Note, that NSs explore large temperatures in core-collapse supernovae and in the postmerger phase of NS mergers. In these scenarios, temperature effects cannot be neglected.
} 


\subsection{Chiral effective field theory}

At low densities, we know that the NS degrees of freedom are nucleonic. In the outer crust, nucleons are contained in increasingly neutron-rich nuclei surrounded by an electron gas, and in the inner crust additionally by a neutron gas. The EOS describing the crust is rather well constrained. Above $0.5 n_{\text {sat }}$, NS matter is composed of a fluid of neutrons, protons, and electrons in $\beta$ equilibrium. Here, microscopic calculations can provide constraints. Such calculations require nuclear Hamiltonians that describe the interactions among nucleons. There have been very successful phenomenological Hamiltonians describing the interactions as a sum of meson-exchanges and short-range structures [10, 11]. However, nuclear interactions are described by infinitely many processes of different importance involving larger numbers of nucleons, pions, or heavier mesons. It is important to find a way of arranging these processes according to their importance, to enable reliable microscopic calculations of the EOS.

Chiral effective field theory (EFT) is a systematic expansion of nuclear forces in which the most general Lagrangian consistent with all symmetries of QCD, containing all processes among nucleons and pions, is expanded in powers of momenta $k$ [12]. The radius of convergence of this expansion is described by the breakdown scale, $\Lambda_{b} \sim 600 \mathrm{MeV}$ [13], which denotes when new d.o.f. need to be explicitly included. In chiral EFT, one- and multi-pion exchanges are explicitly included while shortrange interactions are modeled by a series of contact interactions, which are usually parametrized by certain spin-isospin operators together with low-energy couplings (LECs) that determine their strength. Chiral EFT naturally and consistently includes also important many-nucleon forces. The individual terms in the expansion are arranged according to a power-counting scheme, resulting in an order-by-order theory for nuclear forces that is truncated at a certain order. This allows to construct Hamiltonians with desired accuracy that can be systematically improved by going to higher orders. The LECs are typically fit to nucleon-nucleon scattering data as well as properties of light nuclei. However, new ways of constraining the LECs are currently explored [14]. Importantly, the chiral EFT scheme allows to estimate theoretical uncertainties of the nuclear Hamiltonian due to the truncation of the series. These advantages make chiral EFT the current state-of-the-art for nuclear forces, and make it especially suited when studying systems that are not accessible in experiments, like neutron-rich matter. In the next section, I will present results of quantum Monte Carlo (QMC) calculations [17] using the local chiral interactions of Refs. $[15,16]$. In particular, the results I show have been obtained using the auxiliary-field diffusion Monte Carlo (AFDMC) method [18]. I will not discuss these methods in detail and refer the reader to the reviews of Refs. [17, 19].

\subsection{Results for nuclear systems}

The combination of local chiral interactions with QMC methods has lead to an excellent description of various nuclear systems, from atomic nuclei up to ${ }^{16} \mathrm{O}$ [20], neutron- $\alpha$ scattering [16], as well as pure neutron matter [16, 21]. I show results for binding energies of atomic nuclei up to ${ }^{16} \mathrm{O}$ as well as for neutron matter in Fig. 2; details of these calculations can be found in the respective references. Chiral interactions provide a good description of properties of light nuclei and neutron matter with theoretical uncertainties. It is obvious, that uncertainties grow fast with density due to the eventual breakdown of the theory. This makes chiral interactions only applicable below $2 n_{\text {sat }}$ and not over the whole density range explored in NSs.

\subsection{Neutron-star equation of state}

To address the impact of NS observations on the EOS, we need to extend the microscopic results to all conditions encountered in the NS. First, the neutron-matter results are extrapolated to $\beta$ equilibrium 

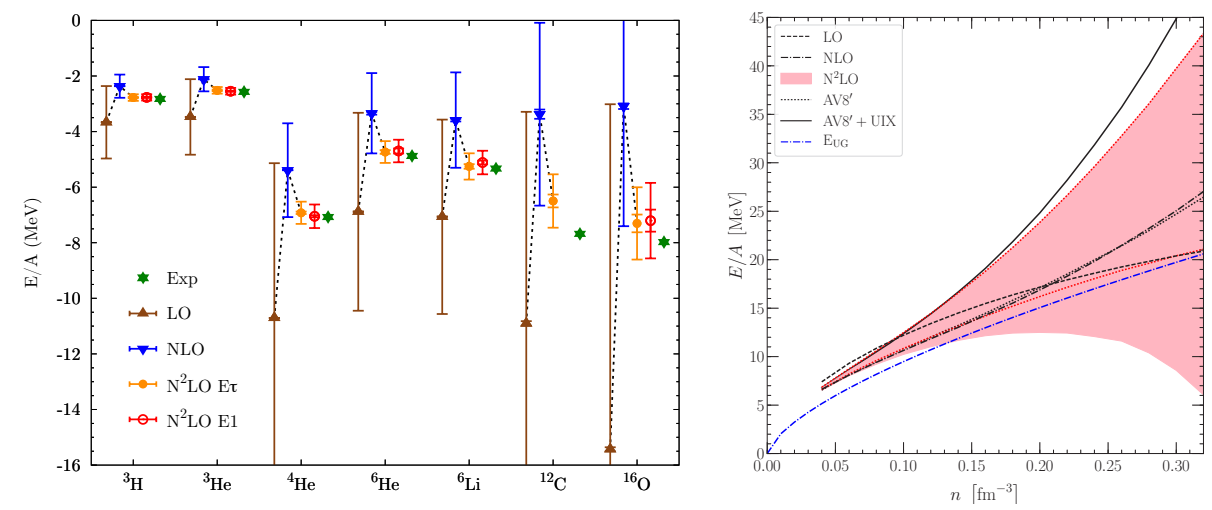

Figure 2. AFDMC results for the binding energies of nuclei up to ${ }^{16} \mathrm{O}$ (left) and for pure neutron matter (right), using local chiral interactions at a cutoff scale $R_{0}=1.0 \mathrm{fm}$. Figures taken from Refs. [20, 22].

and below $0.5 n_{\text {sat }}$ a crust EOS is inserted [23]. The result is the neutron-star EOS constrained up to $2 n_{\text {sat }}$. To reliably extract information from NS observations, it is important to extrapolate to larger densities in a model-agnostic fashion, while not assuming a certain set of degrees of freedom, to reduce systematic uncertainties. Instead, it is important to explore all possibilities for the EOS allowed by general physics considerations, i.e., causality and stability of NSs. Such extension schemes can be based on polytropes [24], or the speed of sound [22]. In the following, we will present results obtained using speed-of-sound extension schemes.

\section{Neutron-star observations and the equation of state}

The EOS extension discussed in the previous section leads to a wide range of NS properties, which can be confronted with observations to improve our knowledge of the EOS.

The first important constraint on the EOS is provided by the observation of heavy NSs. The heaviest NSs known to date have masses of two solar masses [5, 6], which rules out too soft EOS that lead to NSs with small radii and/or small maximum masses. This can be seen in Fig. 3, were the two-solar-mass constraint is indicated by a red-dashed line. This constraint limits the NS radius to be above $\sim 9 \mathrm{~km}$ for a typical $1.4 M_{\odot} \mathrm{NS}$, see the light-gray area.

The next constraint I discuss here comes from the first observation of gravitational waves from a binary NS merger and its electromagnetic counterpart, GW170817 and AT2017gfo [27-29]. The observed gravitational wave signal from the inspiral phase of such NS mergers offers the possibility to measure the NS radius, because it is sensitive to the tidal deformation of the stars. A large star shows a stronger deformation and has a larger tidal deformability $\Lambda$, while a small star is more difficult to deform and has a small $\Lambda$. Because $\Lambda \sim R^{6}$, the determination of the tidal polarizability from GW170817 allows to constrain the radius of a typical NS to be smaller than $13.6 \mathrm{~km}[22,30,31]$.

In addition, the properties of the corresponding observed kilonova allows to further constrain the maximum mass. The amount of ejecta from the kilonova allows to rule out a prompt collapse to a black hole, requiring the EOS to be sufficiently stiff. The energetics of the kilonova on the other hand suggests that no longer-lived NS was formed, which requires the maximum mass to be less than $\sim 2.3 M_{\odot}$ [25]. This produces a narrow range for the maximum mass (indicated in Fig. 3). 

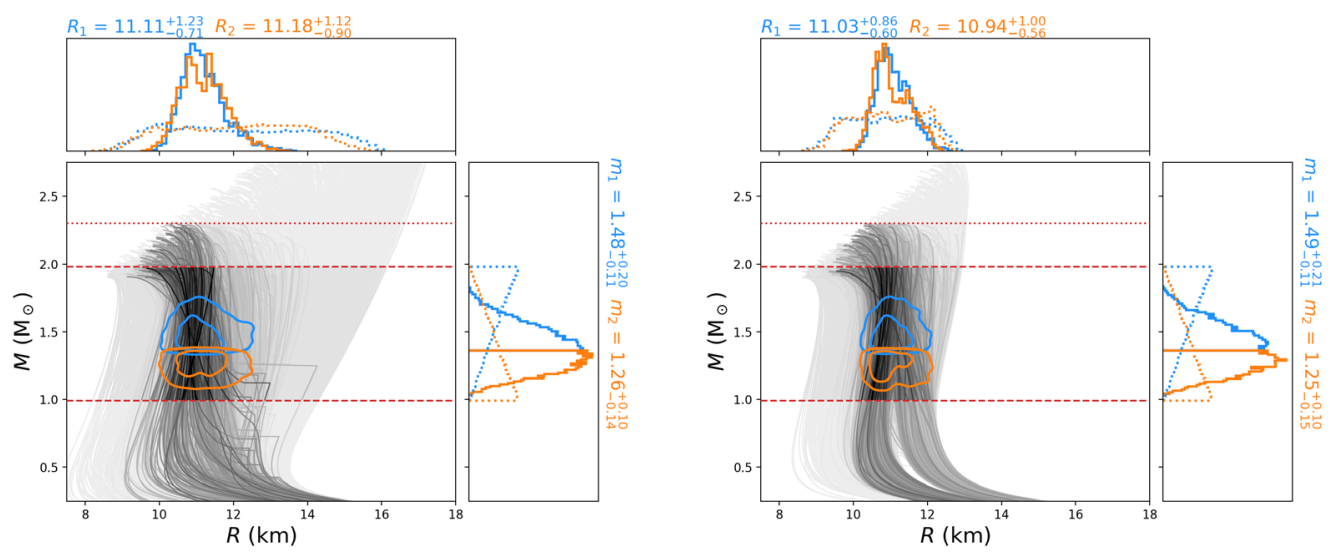

Figure 3. Mass-radius relation of neutron-stars using EOS constrained by chiral EFT up to $n_{\text {sat }}$ (left) or $2 n_{\text {sat }}$ (right) and observational constraints from NS masses, gravitational waves GW170817, and the kilonova observation AT2017gfo. Figure taken from Ref. [26].

A recent reanalysis of the neutron-star merger taking into account EOS constrained by chiral EFT at low densities, the existence of heavy two-solar mass NS, the observed signal GW170817 and the considerations on the maximum mass from the observed kilonova found tight constraints on the NS radius: $R_{1.4}=11.0_{-0.6}^{+0.9} \mathrm{~km}[26]$. We show the corresponding MR relations in Fig. 3 for chiral EFT constraints up to $n_{\text {sat }}$ (left) or up to $2 n_{\text {sat }}$.

\section{Summary and Outlook}

To extract reliable constraints from NS observations, it is necessary to use EOS with reliable uncertainties at low densities and model-agnostic extensions to higher densities to reduce systematic errors. Together with such EOS sets, NS observations offer a unique possibility to probe the EOS of dense nuclear matter at large densities. Current EOS uncertainties are still sizable but will be reduced by improved nuclear-physics constraints at low densities, future observations of NS mergers, and of radii by the NICER mission [32]. This increased knowledge of the NS mass-radius relation might shed light on the possible existence of phase transitions to exotic forms of matter.

I would like to thank the organizers for the invitation and for delivering a successful workshop. I thank D. Brown, S. Brown, C. Capano, J. Carlson, S. De, S. Gandolfi, A. Gezerlis, K. Hebeler, B. Krishnan, S. Kumar, D. Lonardoni, J. Lynn, B. Margalit, J. Margueron, S. Reddy, A. Schwenk for many insightful discussions and their contributions to the studies presented in this work. This work was supported by the U.S. Department of Energy, Office of Science, Office of Nuclear Physics, under Contract No. DE-AC52-06NA25396, by the Los Alamos National Laboratory (LANL) LDRD program, and by the NUCLEI SciDAC program. This research used resources provided by the LANL Institutional Computing Program, which is supported by the U.S. Department of Energy National Nuclear Security Administration under Contract No. 89233218CNA000001. Computational resources have also been provided by the National Energy Research Scientific Computing Center (NERSC), which is supported by the U.S. Department of Energy, Office of Science, under Contract No. DE-AC02-05CH11231, and by the Jülich Supercomputing Center. 


\section{References}

[1] C. L. Fryer, Astrophys. J. 522, 413 (1999).

[2] M. G. Alford, A. Schmitt, K. Rajagopal and T. Schäfer, Rev. Mod. Phys. 80, 1455 (2008).

[3] A. W. Steiner, M. Prakash, J. M. Lattimer, and P. J.Ellis, Phys. Rep. 411, 325 (2005).

[4] J. M. Lattimer, Ann. Rev. Nucl. Part. Sci. 62, 485 (2012).

[5] J. Antoniadis et al., Science 340, 6131 (2013).

[6] H. T. Cromartie et al., Nat. Astron. 4, 72 (2019).

[7] S. Gandolfi, J. Lippuner, A. W. Steiner, I. Tews, X. Du and M. Al-Mamun, J. Phys. G 46, 103001 (2019).

[8] A. Kurkela, P. Romatschke and A. Vuorinen, Phys. Rev. D 81, 105021 (2010).

[9] A. Kurkela, E. S. Fraga, J. Schaffner-Bielich and A. Vuorinen, Astrophys. J. 789, 127 (2014).

[10] R. B. Wiringa, V. G. J. Stoks and R. Schiavilla, Phys. Rev. C 51, 38 (1995).

[11] R. Machleidt, Phys. Rev. C 63, 024001 (2001).

[12] E. Epelbaum, H. W. Hammer and U. G. Meissner, Rev. Mod. Phys. 81, 1773 (2009)

[13] J. A. Melendez, S. Wesolowski and R. J. Furnstahl, Phys. Rev. C 96, no. 2, 024003 (2017)

[14] I. Tews, Z. Davoudi, A. Ekström, J. D. Holt and J. E. Lynn, arXiv:2001.03334 [nucl-th].

[15] A. Gezerlis, I. Tews, E. Epelbaum, M. Freunek, S. Gandolfi, K. Hebeler, A. Nogga and A. Schwenk, Phys. Rev. C 90, 054323 (2014).

[16] J. E. Lynn, I. Tews, J. Carlson, S. Gandolfi, A. Gezerlis, K. E. Schmidt and A. Schwenk, Phys. Rev. Lett. 116, 062501 (2016).

[17] J. Carlson, S. Gandolfi, F. Pederiva, S. C. Pieper, R. Schiavilla, K. E. Schmidt and R. B. Wiringa, Rev. Mod. Phys. 87, 1067 (2015).

[18] K. E. Schmidt and S. Fantoni, Phys. Lett. B 446, 99 (1999).

[19] J. E. Lynn, I. Tews, S. Gandolfi and A. Lovato, Ann. Rev. Nucl. Part. Sci. 69, 279 (2019).

[20] D. Lonardoni, S. Gandolfi, J. E. Lynn, C. Petrie, J. Carlson, K. E. Schmidt and A. Schwenk, Phys. Rev. C 97, 044318 (2018).

[21] I. Tews, J. Carlson, S. Gandolfi and S. Reddy, Astrophys. J. 860, 149 (2018).

[22] I. Tews, J. Margueron and S. Reddy, Eur. Phys. J. A 55, 97 (2019).

[23] I. Tews, Phys. Rev. C 95, 015803 (2017).

[24] K. Hebeler, J. M. Lattimer, C. J. Pethick and A. Schwenk, Astrophys. J. 773, 11 (2013).

[25] M. Shibata, E. Zhou, K. Kiuchi and S. Fujibayashi, Phys. Rev. D 100, no. 2, 023015 (2019)

[26] C. D. Capano et al., arXiv:1908.10352 [astro-ph.HE].

[27] B. P. Abbott et al. [LIGO Scientific and Virgo Collaborations], Phys. Rev. Lett. 119, 161101 (2017).

[28] B. P. Abbott et al., Astrophys. J. 848, 12 (2017).

[29] B. P. Abbott et al. [LIGO Scientific and Virgo Collaborations], Phys. Rev. X 9, 011001 (2019).

[30] E. Annala, T. Gorda, A. Kurkela and A. Vuorinen, Phys. Rev. Lett. 120, 172703 (2018).

[31] E. R. Most, L. R. Weih, L. Rezzolla and J. Schaffner-Bielich, Phys. Rev. Lett. 120, 261103 (2018).

[32] G. Raaijmakers et al., Astrophys. J. Lett. 887, L22 (2019). 